\title{
CVD-Graphene-Based Flexible, Thermoelectrochromic Sensor
}

\author{
Adam Januszko, ${ }^{1}$ Agnieszka Iwan, ${ }^{1}$ Stanislaw Maleczek, ${ }^{1}$ Wojciech Przybyl, ${ }^{1}$ \\ Iwona Pasternak, ${ }^{2}$ Aleksandra Krajewska, ${ }^{2}$ and Wlodzimierz Strupinski ${ }^{2}$ \\ ${ }^{1}$ Military Institute of Engineer Technology, Obornicka 136 Str., 50-961 Wroclaw, Poland \\ ${ }^{2}$ Institute of Electronic Materials Technology, Wolczynska 133 Str., 01-919 Warsaw, Poland \\ Correspondence should be addressed to Adam Januszko; januszko@witi.wroc.pl
}

Received 29 March 2017; Accepted 21 June 2017; Published 27 July 2017

Academic Editor: Stefano Bellucci

Copyright ( 2017 Adam Januszko et al. This is an open access article distributed under the Creative Commons Attribution License, which permits unrestricted use, distribution, and reproduction in any medium, provided the original work is properly cited.

\begin{abstract}
The main idea behind this work was demonstrated in a form of a new thermoelectrochromic sensor on a flexible substrate using graphene as an electrically reconfigurable thermal medium $\left(\mathrm{TEChrom}^{\mathrm{TM}}\right)$. Our approach relies on electromodulation of thermal properties of graphene on poly(ethylene terephthalate) (PET) via mechanical destruction of a graphene layer. Graphene applied in this work was obtained by chemical vapor deposition (CVD) technique on copper substrate and characterized by Raman and scanning tunneling spectroscopy. Electrical parameters of graphene were evaluated by the van der Pauw method on the transferred graphene layers onto $\mathrm{SiO}_{2}$ substrates by electrochemical delamination method. Two configurations of architecture of sensors, without and with the thermochromic layer, were investigated, taking into account the increase of voltage from 0 to $50 \mathrm{~V}$ and were observed by thermographic camera to define heat energy. Current-voltage characteristics obtained for the sensor with damaged graphene layer are linear, and the resistivity is independent from the current applied. The device investigated under $1000 \mathrm{~W} / \mathrm{m}^{2}$ exhibited rise of resistivity along with increased temperature. Flexible thermoelectrochromic device with graphene presented here can be widely used as a sensor for both the military and civil monitoring.
\end{abstract}

\section{Introduction}

Graphene as a very attractive material has been widely tested in several optoelectronic devices, such as solar cells, supercapacitors, light-emitting diodes, displays, touch screens, smart windows, or sensors [1-3]. However, taking into consideration the parameters of the devices constructed with graphene, the methods of graphene preparation strongly impact the performances of these devices [e.g., [3-7]]. Two main methods of graphene synthesis are known: (1) the topdown method (mechanical exfoliation, chemical oxidation, and exfoliation) and (2) the bottom-up method (epitaxial growth on different substrates, chemical vapor deposition (CVD), and arc discharging methods) [6-8]. CVD method of graphene film preparation is much more attractive for the optoelectronic devices due to its superior electrical and surface properties compared to the reduced graphene oxide (rGO) one. CVD graphene is transferable to varied substrates, making the technique multipurpose for many applications.
One such application is as an electrode in an organic and inorganic (silicon) solar cell. CVD-graphene is quasi-twodimensional, since electrons can only move between carbon atoms in the 2D lattice. The lack of a third dimension in CVDgraphene influences various novel properties of this material. For example, one such single mobile charge carrier system is created as an effect of electrons interacting with the carbon atoms in the lattice. The ballistic moves of the carrier over the graphene surface are known, and they allow graphene sheets to conduct electricity very well [9]. Additionally, other various interactions among electrons and the hexagonal lattice make CVD-graphene transparent, flexible, and quite strong [10]. Taking into account the specific properties of graphene and its derivatives, photoluminescence (PL) [11], liquid crystalline (LC) [12], and electrochromic (EC) properties $[2,13]$ are investigated.

However, it is a long way for graphene devices to be used. For this reason, the main goal of this work was the creation of a new thermoelectrochromic sensor on a flexible 


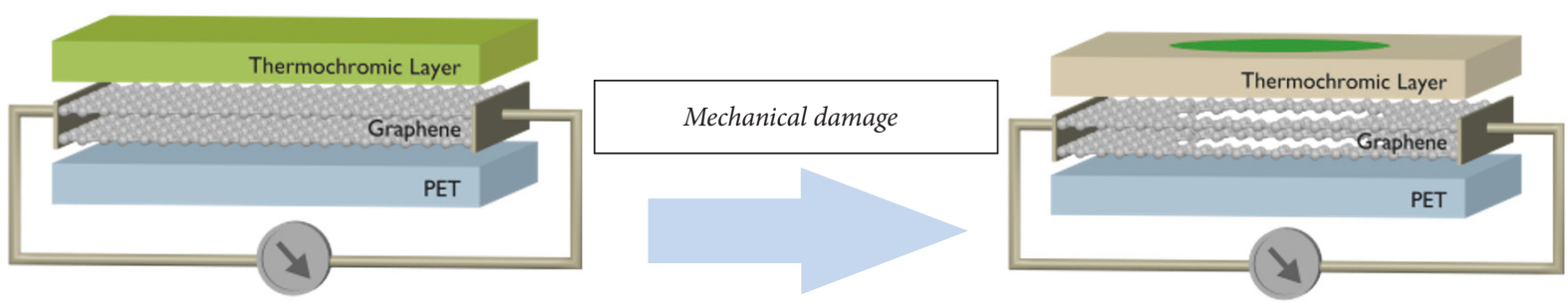

FIGURE 1: CVD-graphene-based thermoelectrochromic sensor on a poly(ethylene terephthalate) (PET) substrate.

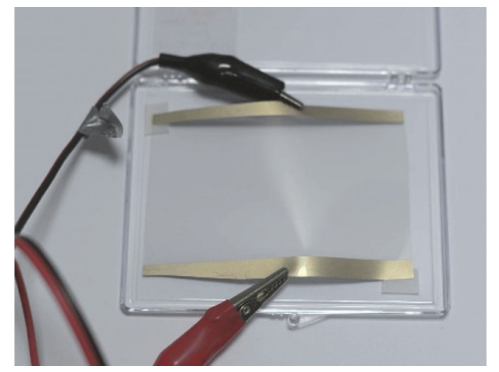

(a)



(b)

FIgURE 2: Photos of (a) CVD-graphene device on the PET substrate and (b) CVD-graphene device on the PET substrate with thermochromic strips.

poly(ethylene terephthalate) (PET) substrate using CVDgraphene as an electrically reconfigurable thermal medium. Schematically, our idea is presented in Figure 1.

In our work, two types of investigations were done and analyzed:

(i) Synthesis and characterization of graphene obtained via CVD method

(ii) Construction of thermoelectrochromic sensors with CVD-graphene and their electrical characterization [14]

Finally, we demonstrated how our simple idea could be effectively exploited in a practical application such as a thermoelectrochromic device on flexible substrate with CVDgraphene layer for monitoring.

\section{Materials and Methods}

2.1. Materials. Multicolor strips with leuco dyes were used as received from the printing office (ETC, Poland).

2.2. Synthesis of CVD-Graphene. Graphene films have been synthesized on copper foils via CVD method using commercially available systems enabling the growth on $\mathrm{Cu}$ substrates, the size of which ranged from $6^{\prime \prime}$ diam. (Aixtron BM Pro) up to $50 \mathrm{~cm} \times 50 \mathrm{~cm}$ (CVD Equipment Corporation, Easy Graphene and GraphMaster Seco/Warwick models). During the thermal process, carbon is liberated from the hydrocarbons and it attaches to the surface of the copper, thus creating graphene rather than precipitating, and segregates from the bulk upon cooling down. Growth on $\mathrm{Cu}$ is also limited largely to one monolayer. Copper acts as a catalyst in the reaction, which suggests that the growth is self-limited.
Carbon adsorbates exhibit high mobility on $\mathrm{Cu}$ surfaces at elevated temperatures, facilitating diffusion across the surface to join the edge of nucleating crystallites. The CVD process starts with the $\mathrm{Cu}$ substrate pretreatment at $\sim 1000^{\circ} \mathrm{C}$ under an Ar gas flow and then $\mathrm{H}_{2}$ gas flow at a pressure of less than 100 mbar. The purpose of this step is to improve the quality and enlarge the grain size of the copper. Afterwards, both the hydrocarbon (e.g., $\mathrm{CH}_{4}$ or $\mathrm{C}_{3} \mathrm{H}_{8}$ ) and the $\mathrm{H}_{2}$ gas are introduced into the reactor for a couple of minutes to grow the graphene layer. Finally, the copper substrate covered with graphene films is cooled down to room temperature in an $\mathrm{Ar}$ atmosphere [15-18].

2.3. Preparation of Thermoelectrochromic Devices. At a first attempt, graphene was transferred from copper foil onto flexible substrate by high-speed electrochemical delamination method [17]. Then flexible CVD-graphene substrate (see Figure 2(a)) was covered with four strips of different colors (see Figure 2(b)). Thermally deposited gold (Au) was used as both electrodes in created CVD-graphene devices.

2.4. Characterization. Graphene grown on Copper foil was investigated using a complementary characterization technique. Raman spectroscopy confirmed the formation of graphitic structures. Moreover, it provided information on the domain size, strain, and stacking order of the graphene films. The morphology of graphene on metal grains was analyzed by scanning electron microscopy (SEM) using Hitachi SU8230 Cold Field Emission system, which was also used to show the topography of the graphene substrate's interface and confirm the uniformity of the graphene layer. Electrical parameters were evaluated by van der Pauw method (using Ecopia HMS-3000 unit) on transferred graphene layers to 




(a)



(b)

FIgURE 3: Optical images of (a) continuous and (b) discontinuous graphene films on Cu substrates.

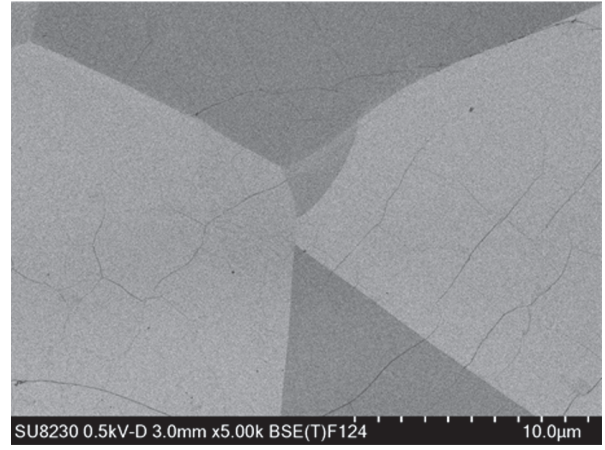

(a)

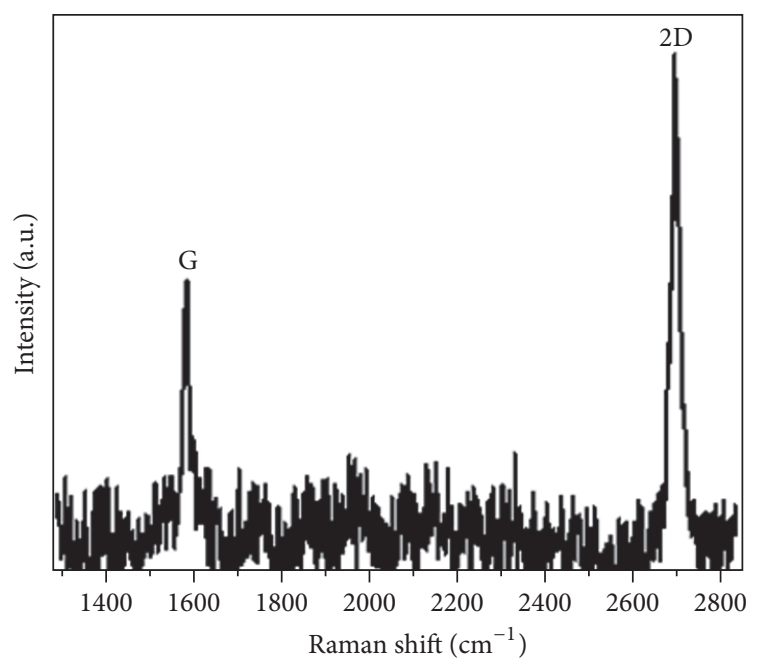

(b)

FIGURE 4: SEM images of graphene layers (a) of high-quality graphene grown on Cu substrate and the typical Raman spectra (b) of graphene layer grown on $\mathrm{Cu}$ substrate.

$\mathrm{SiO}_{2}$ substrates by electrochemical delamination method. The charge carriers' concentration was typically in the range of $1 \times 10^{12}-1 \times 10^{13} / \mathrm{cm}^{2}$, while the Hall mobility at RT was in the level of $2000-4000 \mathrm{~cm}^{2} /$ Vs with $p$-type of conductivity.

Raman spectroscopy (Renishaw's inVia Raman microscope system with a $532 \mathrm{~nm}$ Nd:YAG laser as an excitation source) is normally used to confirm the presence of graphene on the $\mathrm{Cu}$ substrates (see Figure 4(b)). However, optical microscopy with Nomarski contrast is also very useful (Figure 3). This fast and easy measurement exposes cracks and microholes in the graphene layer and allows an analysis of the substrate deformation process, which can affect graphene film's quality.

Measurements done by SEM (see Figure 4(a)) can identify dark spots interpreted as adlayers of graphene (nonexistent in the sample presented). In addition, wrinkles characteristic of graphene grown on a copper substrate are visible.
Electrical properties of both devices with the size of $70 \mathrm{~mm} \times 100 \mathrm{~mm}$ were investigated by laboratory power supply (PWR400L, Kikusui Electronics Corporation, Japan) and universal electric meter (UNI-T UT70A, AXIOMET, Poland). Starting temperature, $T_{0}=25^{\circ} \mathrm{C}$, was the temperature before current application, while $T_{k}=27^{\circ} \mathrm{C}$ was the temperature after current had been applied to the graphene layer, as demonstrated with TESTO 435 (Testo AG, Germany).

A hand-made SOLARBOX (Military Institute of Engineer Technology, Wroclaw, Poland) was used to investigate the electrical behavior of CVD-graphene-based device under $1000 \mathrm{~W} / \mathrm{m}^{2}$ solar irradiation along with increase of temperature from 13 to $30^{\circ} \mathrm{C}$.

Mechanical damage of graphene layer was done via technical calipers, drawing circular patterns at the middle of graphene layer with increasing diameter (from 0 to $60 \mathrm{~mm}$ ), and observed using thermographic camera (VIGOcam v50, VIGO System S.A., Poland). 


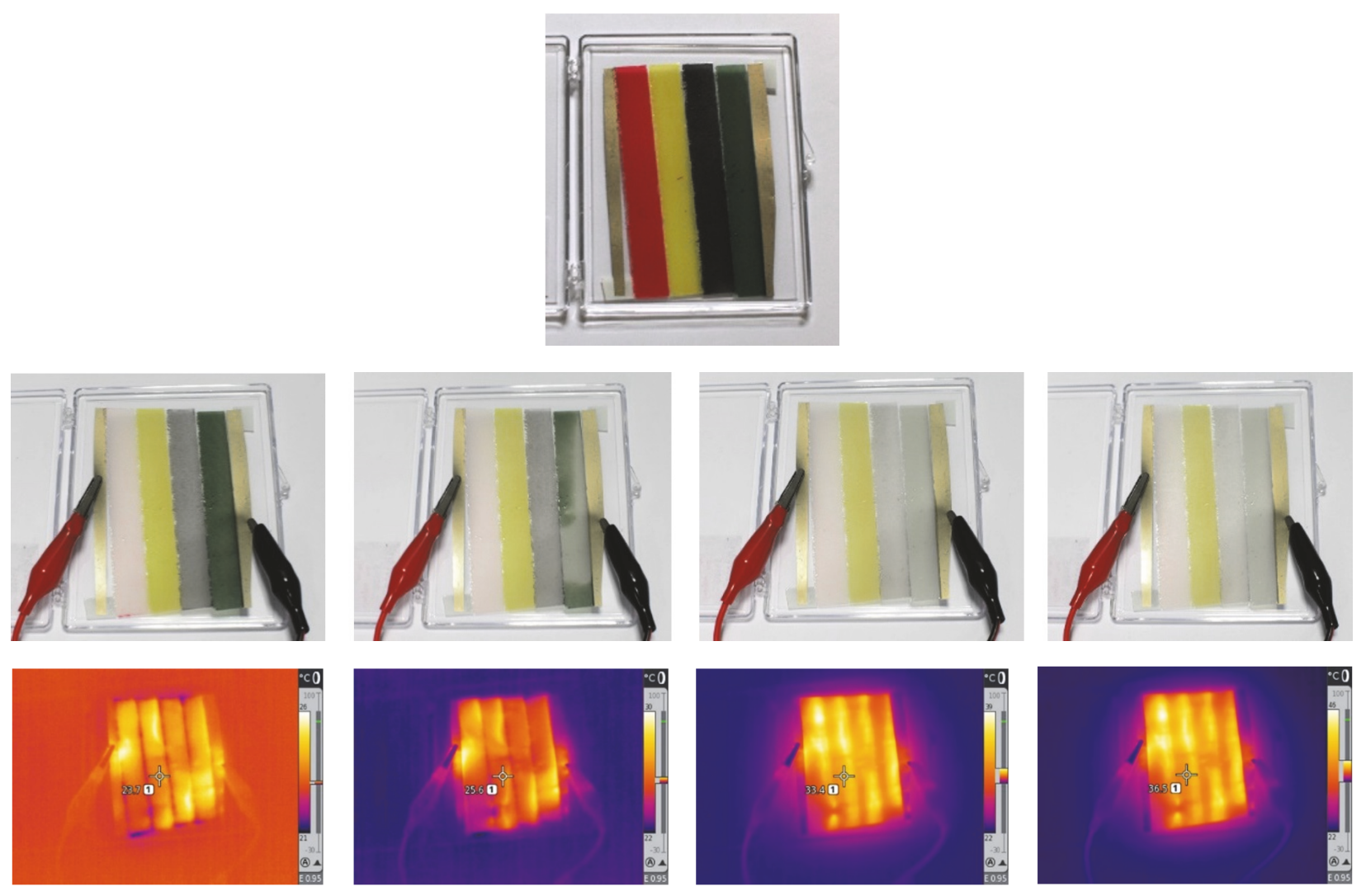

Figure 5: Photos of CVD-graphene devices with thermochromic strips at $0 \mathrm{~V}\left(20^{\circ} \mathrm{C}\right), 10 \mathrm{~V}\left(23.5^{\circ} \mathrm{C}\right), 17.5 \mathrm{~V}\left(26^{\circ} \mathrm{C}\right), 25 \mathrm{~V}\left(33.5^{\circ} \mathrm{C}\right)$, and $30.1 \mathrm{~V}$ $\left(37^{\circ} \mathrm{C}\right)$, respectively, from left to right together with thermal images.

\section{Results and Discussions}

3.1. CVD-Graphene Preparation. Graphene synthesis using CVD method performed on the surface of transition metals is one of the most promising techniques for producing graphene for low-cost applications on a large scale. The precipitation of graphene on bulk transition metals occurs as a result of the temperature-dependent solubility of carbon. The bonding of a single graphene layer to a metal surface is determined by the metal surface itself, the quality of substrate, and the grain size.

\subsection{Thermoelectrochromic Behavior of CVD-Graphene Device} with Thermochromic Strips. At the first stage of our work, we tested the possibility of changing the color of the thermochromic strips as a result of electromodulation of thermal properties of graphene. For this reason, the device was investigated taking into account increase of voltage from 0 to $30 \mathrm{~V}$. As is presented in Figure 5, along with increase of voltage and consequently temperature applied, the multicolor layer changed its color. This behavior clearly confirmed that our idea had worked and thermal properties of electromodulation of CVD-graphene could be used in sensors.

As is presented in Figure 5 along with increase of voltage, change in the color of selected strips was found as a result of increased temperature of graphene layer as is clearly indicated by thermographic camera.
Our CVD-graphene device exhibited reversible effect and is stable in air atmosphere. In our work, used strips are air- and time-stable and exhibited reversible change of color along with variation of the temperature. To explain this effect, we used commercially available Peltier device to change the temperature of the strips as is shown in Figure 6 as an example for the red strip and in Supplementary Material available online at https://doi.org/10.1155/2017/2757590.

The idea behind the study is a practical and efficient use of a $2 \mathrm{D}$ graphene structure in a flexible thermoelectrochromic sensor. The advantage lies in electric properties of graphene which, in this particular solution, are quite favorable: applying voltage to the graphene layer via electrodes results in the increase of this layer's temperature. The application of thermochromic materials in the sensor affects changes of its transparency and/or color.

The electrochromic layer, which had been applied as a cover for the graphene structure and previously had a color, is now colorless (transparent). Layering these layers onto a flexible substrate (base layer) made of PET, for example, enables the sensor to adapt to the surface of the object which is to be monitored.

The resultant changes (damage) can be observed either using a voltage/current meter for current-voltage characteristics, employing a thermal imaging camera, or even with an unaided eye, as the change in color of the thermochromic material is visible. 



FIgURE 6: Photos of red strip along with increase in temperature from 20 and 22 to $24^{\circ} \mathrm{C}$, respectively.
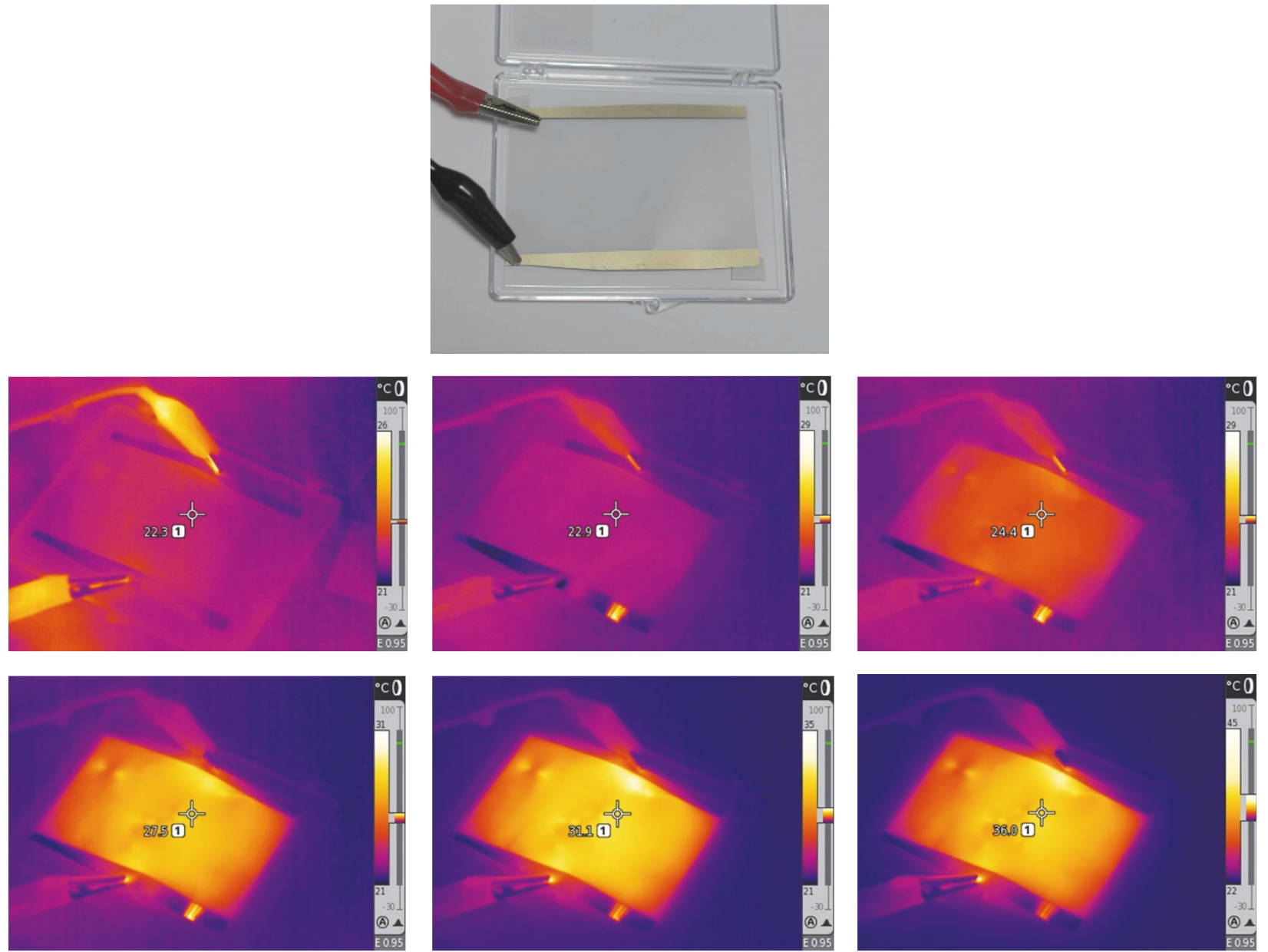

FIGURE 7: Photographs of CVD-graphene device before mechanical damage, together with thermal images at various voltages applied (22.3, $22.9,24.4,27.5,31.1$, and $36 \mathrm{~V}$ from left to right, resp.).

When the voltage is applied, the developed flexible thermoelectrochromic sensor is characterized by two states: 1 , operating, or 0 , damaged. We can differentiate between the two due to the color change and/or change in the transparency.

1, Operating. The device is transparent and does not exhibit any color resulting from a dye contained in the thermochromic layer.

0, Damaged. The device becomes nontransparent due to physiochemical processes within the thermochromic layer which occur due to drop in the temperature of the graphene layer.
To summarize, the invention can be used as a sensor which confirms the presence and the location of damage to, for example, shop window panes, automotive glazing or ballistic covers of helicopters, bulletproof vests, or flak jackets. Thanks to this sensor, the level of safety can be monitored and the safety of the users, such as police officers, soldiers, and ordinary citizens, can be greatly improved.

This structure can serve as an example of combining two unique properties, the electrical properties of graphene layer and optical properties of thermochromic material, which can be applied as an inner layer of glass panes or ballistic covers.

3.3. Electrical Properties of CVD-Graphene-Based Device before and after Mechanical Damage. The main goal of this 

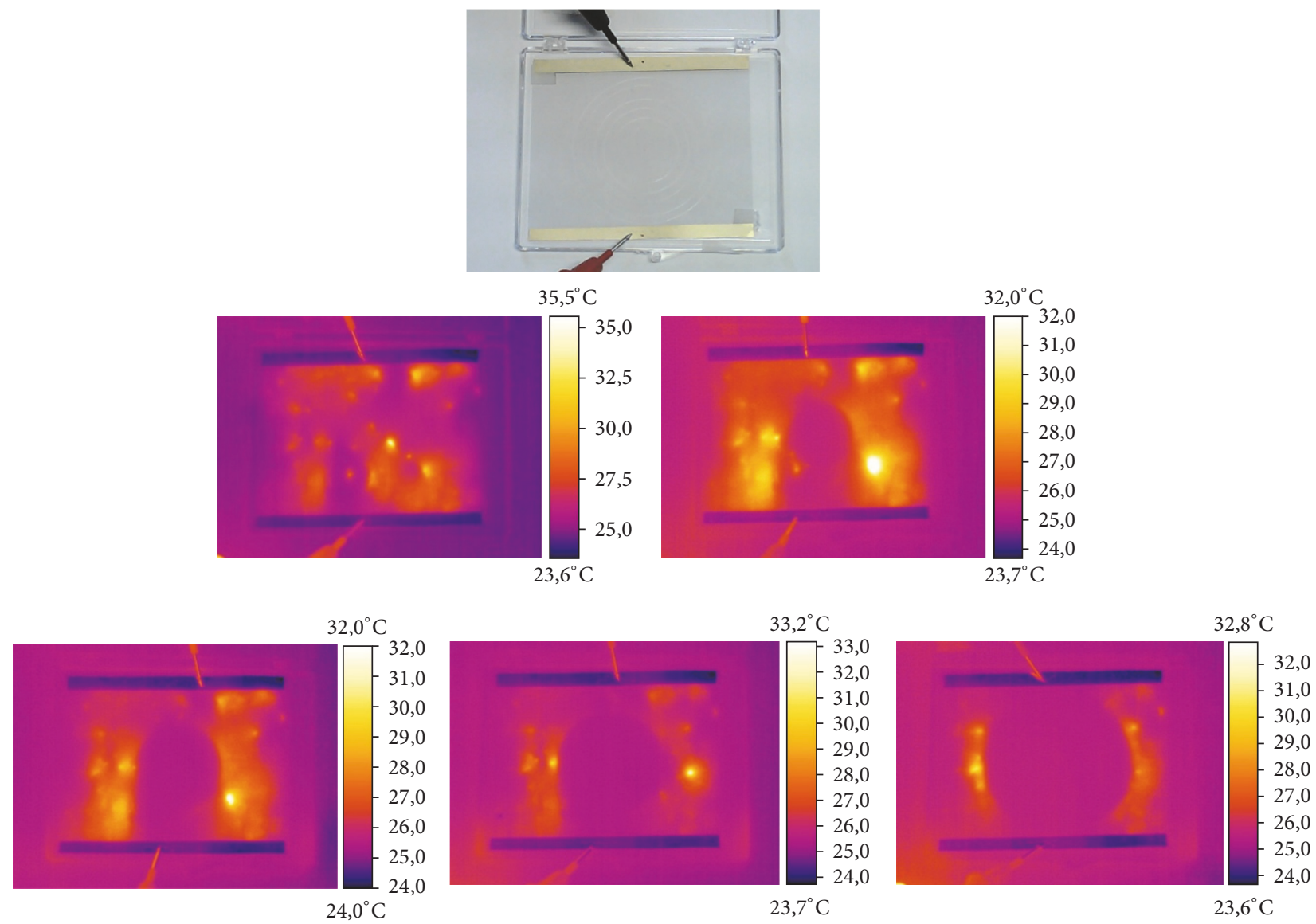

Figure 8: Photos of CVD-graphene device after mechanical damage at $30 \mathrm{~V}$, together with thermal images (diameter size: 0 , 20, 30, 40, and $60 \mathrm{~mm}$, from left to right, resp.).

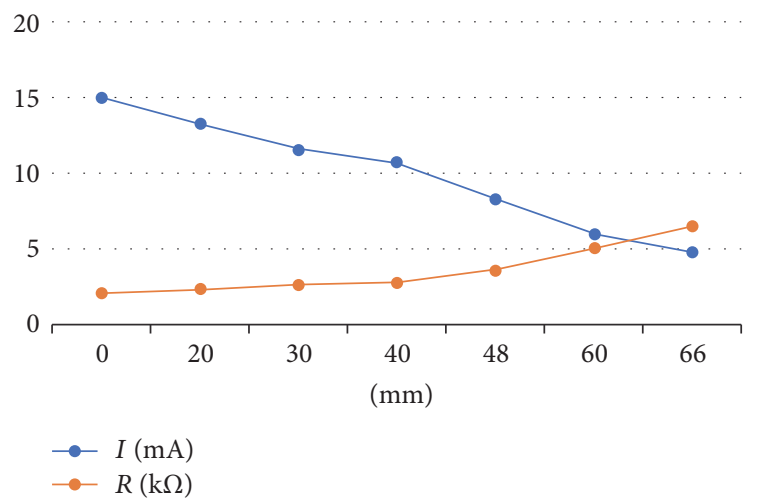

FIGURE 9: Dependence of $I$ and $R$ on the diameter of CVDgraphene-based device.

work was the verification of the proposed CVD-graphenebased device's potential application as sensor to identify mechanical damage. A device with CVD-graphene before and after mechanical damage was investigated by electrical method. In Figures 7 and 8, photographs of CVD-graphenebased devices before and after mechanical scratch are shown together with thermal images.
The device was investigated taking into account increase of voltage from 0 to $50 \mathrm{~V}$. Total degradation of the graphene device was found over $50 \mathrm{~V}$. Electrical properties of the mechanically damaged CVD-graphene layers are presented in Table 1 and in Figure 9.

Our study has shown that, along with increase of the diameter of circle on the graphene layer from 0 to $66 \mathrm{~mm}$ and consequently the increase of mechanical damage to the graphene layer, the value of current $(I)$ decreased from $15 \mathrm{~mA}$ to about $4.7 \mathrm{~mA}$ (see Figure 9). For the CVD-graphene layer before mechanical destruction, we found that, along with the increase of current to $15 \mathrm{~mA}$, the value of voltage increased linearly and resistance $(R)$ was independent from the current applied.

3.4. Electrical Properties of CVD-Graphene-Based Device under $1000 \mathrm{~W} / \mathrm{m}^{2}$ Solar Irradiation. Finally, preliminary test concerning the impact of $1000 \mathrm{~W} / \mathrm{m}^{2}$ solar irradiation on the resistivity of CVD-graphene-based device was investigated along with the increase of temperature from 13 to $30^{\circ} \mathrm{C}$ as an effect of solar irradiation. A hand-made SOLARBOX was used to investigate the electrical behavior of the sensor (see Figure 10).

Our preliminary study shows that, along with increase of temperature from 13 to $30^{\circ} \mathrm{C}$ as an effect of solar irradiation, 
TABLE 1: Electrical properties of CVD-graphene-based device before and after mechanical damage.

\begin{tabular}{|c|c|c|c|c|c|c|c|}
\hline \multirow{2}{*}{ Parameters } & \multicolumn{7}{|c|}{ Diameter $[\mathrm{mm}]$} \\
\hline & 0 & 20 & 30 & 40 & 48 & 60 & 66 \\
\hline$S^{*}\left[\mathrm{~cm}^{2}\right]$ & 0 & 3.1 & 7.1 & 12.6 & 18.1 & 28.3 & 34.2 \\
\hline$I[\mathrm{~mA}]$ & 15.0 & 13.2 & 11.5 & 10.7 & 8.2 & 6.0 & 4.7 \\
\hline$R[\mathrm{k} \Omega]$ & 2.0 & 2.3 & 2.6 & 2.8 & 3.6 & 5.0 & 6.4 \\
\hline
\end{tabular}

${ }^{*}$ Surface area.

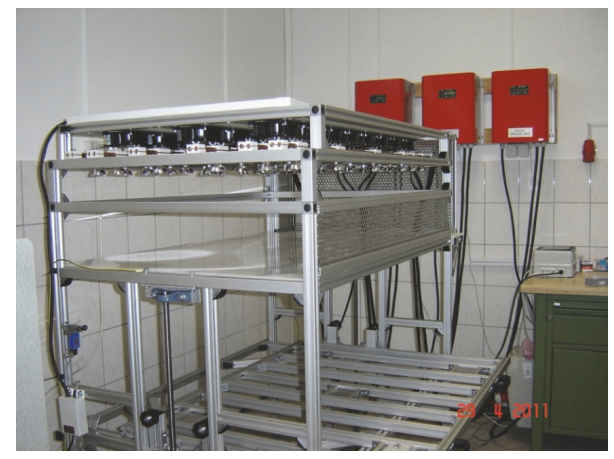

FIGURE 10: SOLARBOX constructed at Military Institute of Engineer Technology, Wroclaw, Poland.

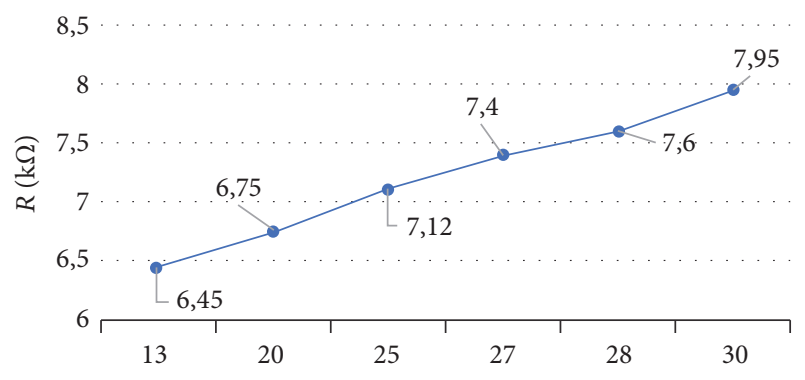

$\left({ }^{\circ} \mathrm{C}\right)$

FIGURE 11: Resistance of CVD-graphene-based sensor under $1000 \mathrm{~W} / \mathrm{m}^{2}$ solar irradiation.

linear increase of resistance $(R)$ for the investigated sensor was found (see Figure 11).

Moreover, we calculated a temperature resistance coefficient $(\alpha)$ of graphene sensor based on the equation $\alpha=$ $\left(R_{T} / R_{o}\right)-1 /\left(T-T_{o}\right)$. The value obtained equaled $13.6 \times$ $10^{-3}\left[\mathrm{~K}^{-1}\right]$ and it is few times higher than, for example, that for $\mathrm{Cu}\left(3.9 \times 10^{-3}\left[\mathrm{~K}^{-1}\right]\right)$. Based on the preliminary study, we can conclude that the developed CVD-graphene-based device can be applied as a sensor for temperature and detection of mechanical defects. Moreover, taking into account other practical applications of graphene, our experiment provides first feedback on graphene being applied as electrode or in an encapsulated form as a layer in solar cells.

\section{Conclusions}

New thermoelectrochromic sensor (TEChrom) on flexible substrate was constructed using CVD-graphene layers. We showed that the electrical properties of the investigated sensor can be controlled by change in temperature, area of mechanical destruction of sensor, and time of solar irradiation. The main advantage of our flexible sensor is the simplicity of the device architecture. CVD technique applied in order to create graphene layers in the sensor gave us the possibility to obtain high-quality graphene and potentially, on a large scale, use it in flexible solar cells integrated with textiles or buildings. The presented flexible thermoelectrochromic device with graphene can be widely used as a sensor for both the military and civil monitoring.

\section{Conflicts of Interest}

The authors declare that there are no conflicts of interest regarding the publication of this paper.

\section{Acknowledgments}

The authors express their gratitude to the National Centre for Research and Development and the National Fund for Environmental Protection and Water Management (no. GEKON2/O4/268473/23/2016) for supplementary financial support of the project. This work was performed within the statute funds of Military Institute of Engineer Technology in 2016 received from the Ministry of Science and Higher Education (no. 15166).

\section{References}

[1] R. Gupta, K. D. M. Rao, S. Kiruthika, and G. U. Kulkarni, "Visibly transparent heaters," ACS Applied Materials and Interfaces, vol. 8, no. 20, pp. 12559-12575, 2016.

[2] E. O. Polat, O. Balc, and C. Kocabas, "Graphene based flexible electrochromic devices," Scientific Reports, vol. 4, article 6484, 2014.

[3] A. Iwan and A. Chuchmała, "Perspectives of applied graphene: polymer solar cells," Progress in Polymer Science, vol. 37, no. 12, pp. 1805-1828, 2012.

[4] N. G. Sahoo, Y. Pan, L. Li, and S. H. Chan, "Graphene-based materials for energy conversion," Advanced Materials, vol. 24, no. 30, pp. 4203-4210, 2012.

[5] Z. Pan, H. Gu, M-T. Wu, Y. Li, and Y. Chen, "Graphene-based functional materials for organic solar cells," Optical Materials Express, vol. 2, pp. 815-824, 2012.

[6] X. Wan, Y. Huang, and Y. Chen, "Focusing on energy and optoelectronic applications: a journey for graphene and graphene oxide at large scale," Accounts of Chemical Research, vol. 45, no. 4, pp. 598-607, 2012. 
[7] Z.-S. Wu, G. Zhou, L.-C. Yin, W. Ren, F. Li, and H.-M. Cheng, "Graphene/metal oxide composite electrode materials for energy storage," Nano Energy, vol. 1, no. 1, pp. 107-131, 2012.

[8] D. R. Dreyer, S. Park, C. W. Bielawski, and R. S. Ruoff, "The chemistry of graphene oxide," Chemical Society Reviews, vol. 39, no. 1, pp. 228-240, 2010.

[9] A. A. K. Geim and K. S. Novoselov, "The rise of graphene," Nature Materials, vol. 6, no. 3, pp. 183-191, 2007.

[10] J. S. Bunch, S. S. Verbridge, J. S. Alden et al., "Impermeable atomic membranes from graphene sheets," Nano Letters, vol. 8, no. 8, pp. $2458-2462,2008$.

[11] D. Pan, J. Zhang, Z. Li, and M. Wu, "Hydrothermal route for cutting graphene sheets into blue-luminescent graphene quantum dots," Advanced Materials, vol. 22, no. 6, pp. 734-738, 2010.

[12] H. K. Bisoyi and S. Kumar, "Carbon-based liquid crystals: art and science," Liquid Crystals, vol. 38, no. 11-12, pp. 1427-1449, 2011.

[13] Z. Ji, S. K. Doorn, and M. Sykora, "Electrochromic graphene molecules," ACS Nano, vol. 9, no. 4, pp. 4043-4049, 2015.

[14] A. Januszko, W. Przybył, A. Krajewska, I. Pasternak, A. Przewloka, and W. Strupinski, Patent application P.413693, 2015.

[15] L. Misseeuw, A. Krajewska, I. Pasternak et al., "Optical-quality controllable wet-chemical doping of graphene through a uniform, transparent and low-roughness F4-TCNQ/MEK layer," RSC Advances, vol. 6, no. 106, pp. 104491-104501, 2016.

[16] I. Pasternak, A. Krajewska, K. Grodecki, I. Jozwik-Biala, K. Sobczak, and W. Strupinski, "Graphene films transfer using marker-frame method," AIP Advances, vol. 4, no. 9, Article ID 097133, 2014.

[17] T. Ciuk, I. Pasternak, A. Krajewska et al., "Properties of chemical vapor deposition graphene transferred by high-speed electrochemical delamination," Journal of Physical Chemistry C, vol. 117, no. 40, pp. 20833-20837, 2013.

[18] I. Wlasny, P. Dabrowski, M. Rogala et al., "Role of graphene defects in corrosion of graphene-coated $\mathrm{Cu}(111)$ surface," Applied Physics Letters, vol. 102, no. 11, Article ID 111601, 2013. 



\section{Hindawi}

Submit your manuscripts at

https://www.hindawi.com
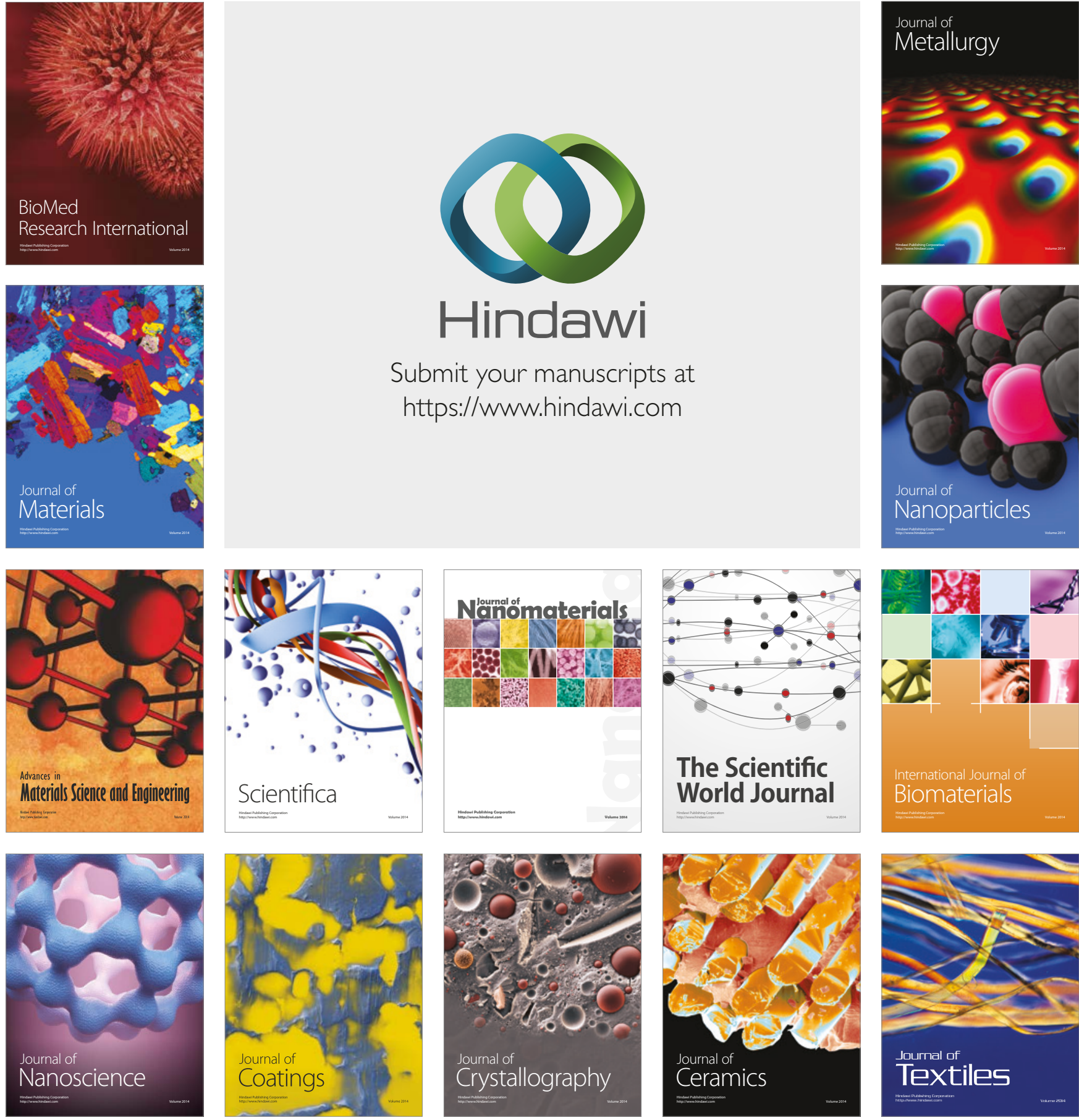



The Scientific World Journal
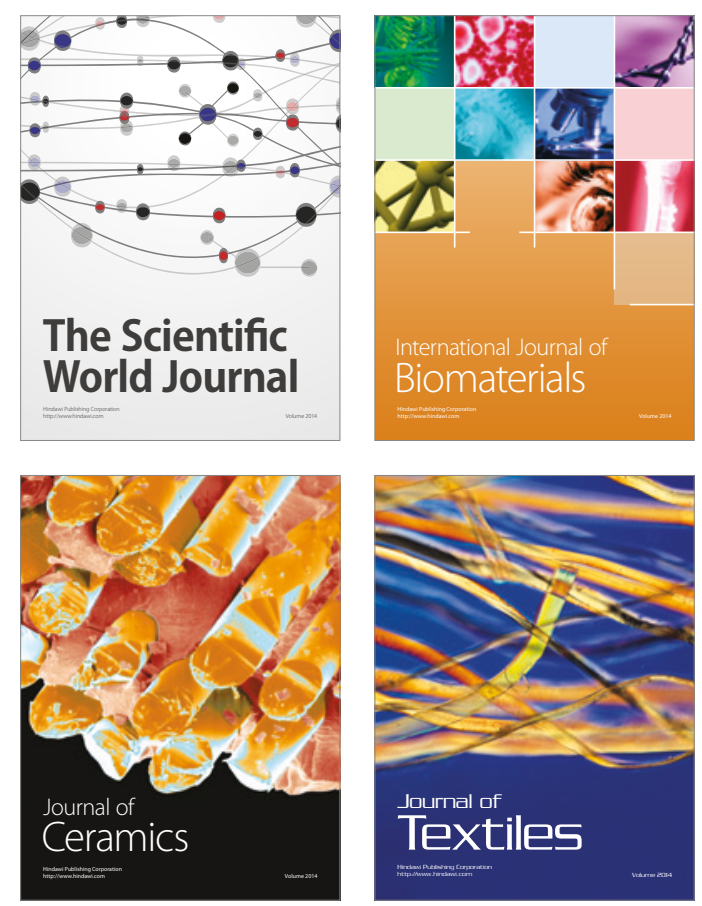